\title{
Temperatures of Exploding Nuclei
}

V. Serfling, ${ }^{1}$ C. Schwarz, ${ }^{1}$ R. Bassini, ${ }^{2}$ M. Begemann-Blaich, ${ }^{1}$ S. Fritz, ${ }^{1}$ S. J. Gaff, ${ }^{3}$ C. Groß,${ }^{1}$ G. Immé, ${ }^{4}$ I. Iori, ${ }^{2}$ U. Kleinevoß, ${ }^{1}$ G. J. Kunde, ${ }^{3, *}$ W. D. Kunze, ${ }^{1}$ U. Lynen, ${ }^{1}$ V. Maddalena,${ }^{4, \dagger}$ M. Mahi, ${ }^{1}$ T. Möhlenkamp, ${ }^{5,}$ A. Moroni, ${ }^{2}$ W. F. J. Müller, ${ }^{1}$ C. Nociforo, ${ }^{4}$ B. Ocker, ${ }^{6}$ T. Odeh, ${ }^{1}$ F. Petruzzelli, ${ }^{2}$ J. Pochodzalla, ${ }^{7}$ G. Raciti, ${ }^{4}$ G. Riccobene,${ }^{4}$ F. P. Romano, ${ }^{4}$ A. Saija, ${ }^{4}$ M. Schnittker, ${ }^{1}$ A. Schüttauf, ${ }^{6}$ W. Seidel,${ }^{5}$ C. Sfienti, ${ }^{4}$ W. Trautmann, ${ }^{1}$ A. Trzcinski, ${ }^{8}$ G. Verde, ${ }^{4}$ A. Wörner, ${ }^{1}$ Hongfei Xi ${ }^{1, \dagger}$ and B. Zwieglinski ${ }^{8}$

${ }^{1}$ Gesellschaft für Schwerionenforschung, D-64291 Darmstadt, Germany

${ }^{2}$ Istituto di Scienze Fisiche, Università degli Studi di Milano and I.N.F.N., I-20133 Milano, Italy

${ }^{3}$ Department of Physics and Astronomy and National Superconducting Cyclotron Laboratory, Michigan State University, East Lansing, Michigan 48824

${ }^{4}$ Dipartimento di Fisica dell' Università and I.N.F.N., I-95129 Catania, Italy

${ }^{5}$ Forschungszentrum Rossendorf, D-01314 Dresden, Germany

${ }^{6}$ Institut für Kernphysik, Universität Frankfurt, D-60486 Frankfurt, Germany

${ }^{7}$ Max-Planck-Institut für Kernphysik, D-69117 Heidelberg, Germany

${ }^{8}$ Soltan Institute for Nuclear Studies, 00-681 Warsaw, Hoza 69, Poland

(Received 17 December 1997)

Breakup temperatures in central collisions of ${ }^{197} \mathrm{Au}+{ }^{197} \mathrm{Au}$ at bombarding energies $E / A=50$ to $200 \mathrm{MeV}$ were determined with two methods. Isotope temperatures, deduced from double ratios of hydrogen, helium, and lithium isotopic yields, increase monotonically with bombarding energy from 5 to $12 \mathrm{MeV}$, in qualitative agreement with a scenario of chemical freeze-out after adiabatic expansion. Excited-state temperatures, derived from yield ratios of states in ${ }^{4} \mathrm{He},{ }^{5,6} \mathrm{Li}$, and ${ }^{8} \mathrm{Be}$, are about $5 \mathrm{MeV}$, independent of the projectile energy, and seem to reflect the internal temperature of fragments at their final separation from the system. [S0031-9007(98)05934-1]

PACS numbers: 25.70.Pq, 21.65.+f, 25.70.Mn, 25.75.Ld

Recently, a caloric curve of nuclei has been obtained by correlating the values of temperature and excitation energy measured for spectator fragmentation in reactions of ${ }^{197} \mathrm{Au}+{ }^{197} \mathrm{Au}$ at $600 \mathrm{MeV}$ per nucleon [1]. The temperatures were derived from double ratios of helium and lithium isotopic yields while the excitation energies were obtained by adding up the kinetic energies of the product nuclei and the mass excess of the observed partition with respect to the ground state of the reconstructed spectator nucleus. The double-bended shape of the caloric curve and its similarity to predictions of microscopic statistical models [2-4], has stimulated considerable experimental and theoretical activities. In particular, the second rise of the temperature to values exceeding $10 \mathrm{MeV}$ at high excitation energies has initiated the discussion of whether nuclear temperatures of this magnitude can be measured reliably (see Refs. [4-6], and references given in these recent papers) and whether this observation may indeed be linked to a transition towards the vapor phase $[7,8]$. Obviously, a well-founded understanding of the significance of the employed temperature observables [9] is indispensable when searching for signals of the predicted liquid-gas phase transition in nuclear matter.

Here, we present the results of temperature measurements for central collisions of ${ }^{197} \mathrm{Au}+{ }^{197} \mathrm{Au}$ at incident energies $E / A=50$ to $200 \mathrm{MeV}$. These collisions are characterized by a collective radial flow of light particles and fragments which, over the covered energy range, evolves as a dynamical phenomenon closely connected to the initial stages of the reaction [10]. Global equilibrium is clearly not achieved. If local equilibrium is reached, the associated temperatures should reflect the adiabatic cooling of the rapidly expanding system.

Two temperature observables were used simultaneously. Isotope temperatures were deduced from double ratios of isotopic yields [11] and excited-state temperatures were derived from the correlated yields of lightparticle coincidences $[9,12,13]$. It will become evident from the diverging results that this represents more than a methodical test and that the two types of thermometers are sensitive to different stages of the fragment formation and emission.

Beams of ${ }^{197} \mathrm{Au}$ with $E / A=50,100,150$, and $200 \mathrm{MeV}$, provided by the heavy-ion synchrotron SIS, were directed onto targets of $75 \mathrm{mg} / \mathrm{cm}^{2}$ areal density. Two multidetector hodoscopes, consisting of 96 and of $64 \mathrm{Si}-\mathrm{CsI}(\mathrm{Tl})$ telescopes in closely packed geometries, were placed on opposite sides with respect to the beam axis. Four high-resolution telescopes [4] were used to measure the isotopically resolved yields of light charged particles and fragments. The choice of angles, between $\theta_{\text {lab }}=24^{\circ}$ and $58^{\circ}$ for the hodoscopes and $\theta_{\text {lab }} \approx 40^{\circ}$ for the telescopes, was motivated by the aim of a good coverage at midrapidity.

Additional detectors were employed in order to probe the charged-particle multiplicity for impact parameter selection. The angular range of $\theta_{\text {lab }}=6^{\circ}$ to $20^{\circ}$ was covered by an azimuthally symmetric array of $36 \mathrm{CaF}_{2}$ plastic phoswich detectors [14]. Within the angular range $30^{\circ}$ to $55^{\circ}$, a solid angle of $0.7 \mathrm{sr}$ was covered by an array 
of 48 elements of Si-strip detectors. The results presented in the following were obtained after selecting an event class of highest associated multiplicity, corresponding to about $10 \%$ of the total reaction cross section.

The populations of particle-unstable resonances were derived from two-particle-coincidence yields measured with the Si-CsI hodoscopes. The peak structures were identified by using the technique of correlation functions, and background corrections were based on results obtained for resonance-free pairs of fragments with $Z \leq 3$, such as $p-d, d-d$, up to ${ }^{3} \mathrm{He}-{ }^{7} \mathrm{Li}$. Examples of correlation functions constructed for $p-{ }^{4} \mathrm{He}$ and $d-{ }^{3} \mathrm{He}$ coincidences are shown in Fig. 1. They are dominated by the resonances corresponding to the ground state (g.s.) and 16.66 MeV excited state of ${ }^{5} \mathrm{Li}$. This pair of states represents a widely used thermometer for nuclear reactions $[12,13,15]$. The observed weak peak intensities are expected for large source sizes and may indicate breakup densities considerably below normal nuclear density [16]. Correlated yields of $p-t, d-{ }^{4} \mathrm{He},{ }^{4} \mathrm{He}-{ }^{4} \mathrm{He}$, and $p-{ }^{7} \mathrm{Li} \mathrm{co}-$ incidences and ${ }^{4} \mathrm{He}$ singles yields were also measured and used to deduce temperatures from the populations of states in ${ }^{4} \mathrm{He}$ (g.s.; group of three states at $20.21 \mathrm{MeV}$ and higher), ${ }^{6} \mathrm{Li}$ (2.19 MeV; group of two states at 4.31 and $5.65 \mathrm{MeV}$ ), and ${ }^{8} \mathrm{Be}$ (g.s.; $3.04 \mathrm{MeV}$; group of five states at $17.64 \mathrm{MeV}$ and higher). The probabilities for the coincident detection of the decay products of these resonances were calculated with a Monte Carlo model $[13,17]$. The uncertainty of the background subtraction is the main contribution to the errors of the deduced temperatures.

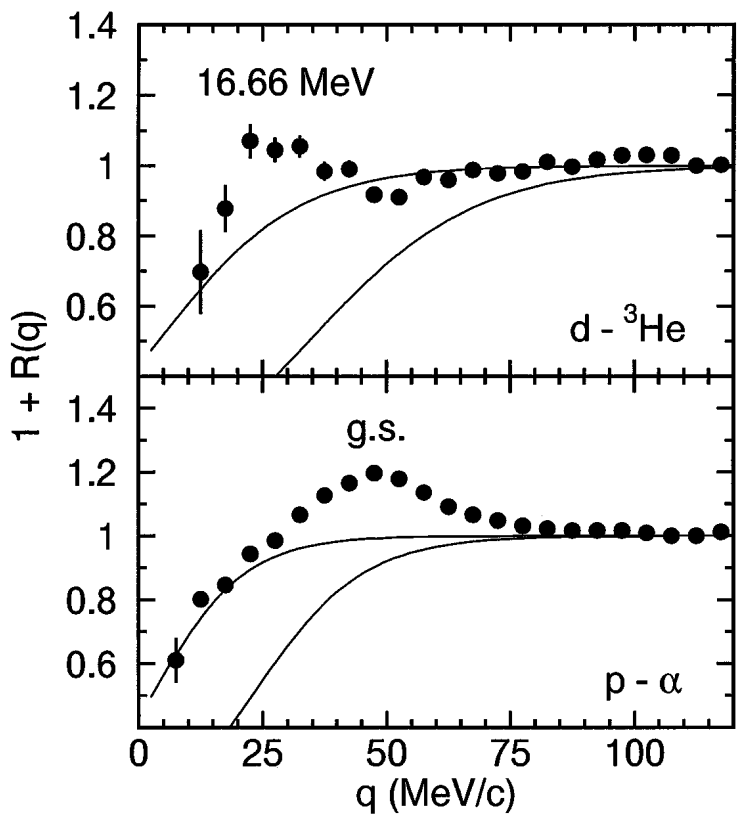

FIG. 1. Correlation functions constructed for $d-{ }^{3} \mathrm{He}$ (top) and $p-{ }^{4} \mathrm{He}$ (bottom) coincidences as a function of the relative momentum $q$ of the two particles for central collisions at $E / A=150 \mathrm{MeV}$. The observed states in ${ }^{5} \mathrm{Li}$ are indicated. The lines represent the adopted upper and lower bounds of the nonresonant background (see text).
The obtained values for two isotope and three excitedstate temperatures are given in Fig. 2. The isotope temperatures $T_{\mathrm{HeLi}}$ and $T_{\text {Hedt }}$ were derived as described in [4], and correction factors of 1.2 have been applied in order to account for the effects of sequential feeding. The three excited-state temperatures are characterized by large energy differences of the considered states (for $T_{\mathrm{Be} 8}$ the $18 \mathrm{MeV} / 3.04 \mathrm{MeV}$ result is shown), and no corrections for sequential feeding were applied (fully justified only for ${ }^{5} \mathrm{Li}$; see below and $\left.[12,15]\right)$. At $E / A=50 \mathrm{MeV}$, all temperature values coincide within the interval $T=4$ to $6 \mathrm{MeV}$, an observation made also at $E / A=35 \mathrm{MeV}$ by Huang et al. [18]. With increasing bombarding energy, however, the isotope temperatures rise approximately linearly up to $T_{\mathrm{HeLi}} \approx 12 \mathrm{MeV}$ and $T_{\mathrm{Hedt}} \approx 9 \mathrm{MeV}$ at $E / A=200 \mathrm{MeV}$. The excited-state temperatures, on the other hand, mutually consistent with each other, appear to be virtually independent of the bombarding energy. Their mean values, over the covered range of bombarding energies, are $4.6 \pm 0.6 \mathrm{MeV}, 5.1 \pm 0.3 \mathrm{MeV}$, and $6.1 \pm$ $0.7 \mathrm{MeV}$ for $T_{\mathrm{Li} 5}, T_{\mathrm{He} 4}$, and $T_{\mathrm{Be} 8}$, respectively. These differences may be significant but seem marginal in comparison to the apparent qualitative difference between the isotope and excited-state temperatures [19].

The momentum-space acceptance of the detectors, kept at fixed positions in the laboratory, changes with bombarding energy in the center-of-mass frame. For the case of $E / A=150 \mathrm{MeV}$, the acceptance of the 96-element hodoscope for $p$ - $\alpha$ coincidences in the momentum interval corresponding to the ${ }^{5} \mathrm{Li}-\mathrm{g}$.s. resonance is shown in Fig. 3 (top). It covers the region around $\theta_{\mathrm{cm}}=90^{\circ}$ and,

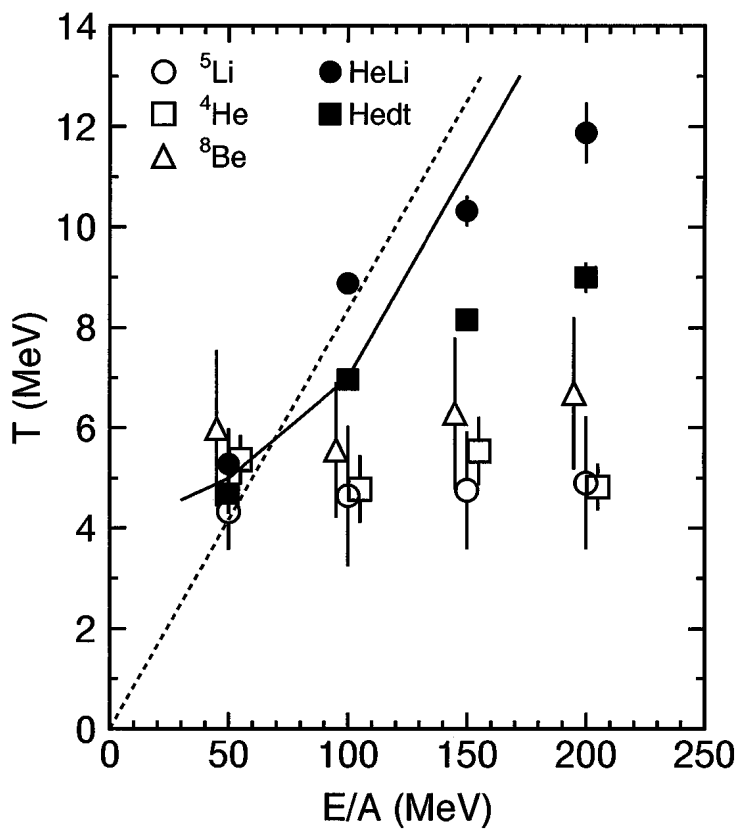

FIG. 2. Measured isotope temperatures (full symbols) and excited-state temperatures (open symbols) as a function of the incident energy per nucleon. The indicated uncertainties are mainly of systematic origin. The meaning of the lines is explained in the text. 

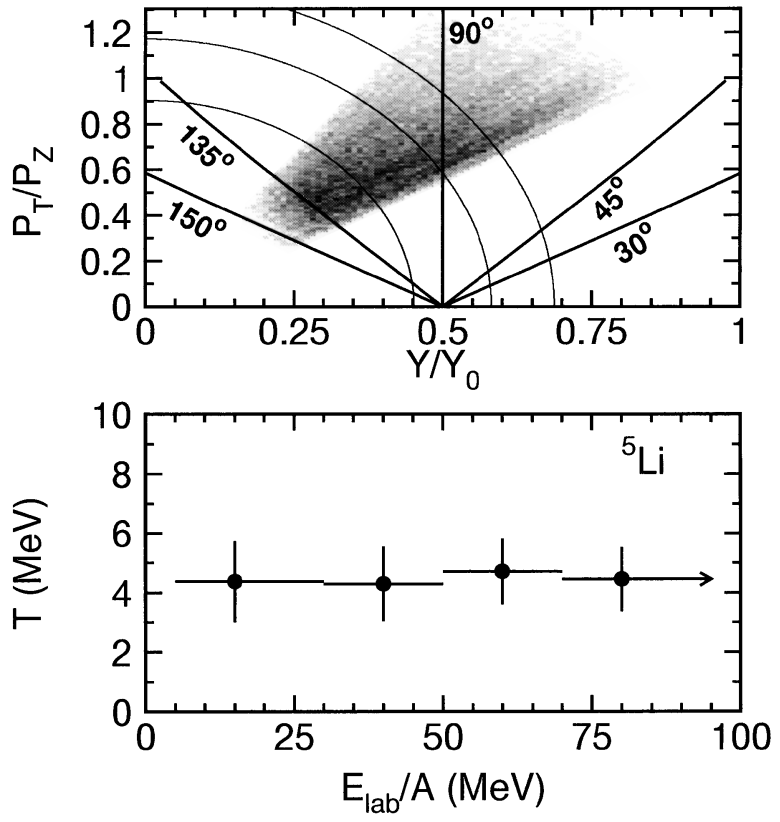

FIG. 3. Top: Acceptance of the 96-element hodoscope for $p-{ }^{4} \mathrm{He}$ coincidences with relative momenta $25 \leq q \leq 75 \mathrm{MeV} / c$ in the plane subtended by relative transverse momentum and normalized rapidity of the corresponding ${ }^{5} \mathrm{Li}$ fragment for $E / A=150 \mathrm{MeV}$. The loci of $\Theta_{\mathrm{cm}}=30^{\circ}$, $45^{\circ}, 90^{\circ}, 135^{\circ}$, and $150^{\circ}$ (thick lines) and of ${ }^{5} \mathrm{Li}$ energies $E_{\mathrm{lab}} / A=30,50$, and $70 \mathrm{MeV}$ (thin lines) are indicated. Bottom: Temperatures derived from the yields of ${ }^{5} \mathrm{Li}$ ground and excited states for the four intervals of the ${ }^{5} \mathrm{Li}$ laboratory energy. Note that the errors are mainly of systematic origin.

in addition, extends to forward and backward angles with a varying transverse-momentum acceptance. The wide acceptance and its shift with bombarding energy should not be crucial, however, because no significant variation of $T_{\mathrm{Li} 5}$ within the covered momentum space was found (Fig. 3, bottom).

It is not immediately obvious that the divergence of the isotope and excited-state temperatures, growing dramatically with bombarding energy, contradicts the concept of a common fragment freeze-out at a single temperature. $\mathrm{Xi}$ et al. report that their recent statistical calculations indicate a strongly reduced sensitivity of the helium-lithium thermometer at high temperature, such that it may prevent reliable temperature measurements at $T>7 \mathrm{MeV}$ [5]. Accordingly, a consistent common temperature, if existing, should be low. The excluded-volume effect, as incorporated in the quantum-statistical model by Gulminelli and Durand [6], causes a suppression of particle-unstable resonances decaying into loosely bound products, such as the $16.66-\mathrm{MeV}$ excited state of ${ }^{5} \mathrm{Li}$. It will have the effect that the apparent $T_{\mathrm{Li} 5}$ is low while a common emission temperature may be high. These calculations demonstrate that large effects can be caused by sequential decay and by structural differences of the nuclear states employed in the temperature measurements, even though they may not suffice to give a consistent explanation of all the present observations.
The dynamical evolution of the fragment formation has very recently been investigated with transport models [20], including nuclear molecular-dynamics [21,22] and quantum molecular-dynamics [23,24] models, applied to the present and similar reactions. These studies suggest that the asymptotic fragments can be identified at an early stage of the reaction, typically at $\approx 40 \mathrm{fm} / c$. These times coincide with the development of the collective flow component of the fragment motion [20-22]. If local chemical equilibrium has been reached the isotopic composition should reflect the temperature of the system at that particular time.

According to various flow analyses, between $40 \%$ and $60 \%$ of the collision energy is converted into collective flow energy [10]. In the simplest approximation, the breakup temperature is then estimated as $T=(E / A) / 12$. This assumes complete stopping of the incident nuclei and a classical gas with $3 \times 2 A$ degrees of freedom carrying a thermal energy component of $50 \%$ of the collision energy. This relation (dashed line in Fig. 2) does not describe the data very well, but it illustrates the expected linear rise and shows that the measured isotope temperatures have about the right order of magnitude. Better agreement with the data at the lower energies is obtained if, for the same thermal energies, the experimental temperature vs energy relation of Ref. [1] is used (Fig. 2, full line). Even though it remains to be understood why $T_{\text {Hedt }}$ is considerably lower in the present case (cf. [4,25-27]), the comparison suggests that the isotope thermometers are sensitive to the local temperature at freeze-out in a blast scenario [28-30].

The excited states used for the temperature evaluation are very specific quantum states with widths of $1 \mathrm{MeV}$ or less. They are unlikely to exist in the nuclear medium in identical forms [20,31,32]. The observed asymptotic states can develop or survive only at very low densities that may not be reached before the cluster is emitted into vacuum. Accordingly, the excited-state populations should reflect the temperature and its fluctuations at this final stage of fragment emission. The molecular dynamics calculations show that a cluster continues to interact with the surrounding cooling and expanding matter for a considerable time after it has been formed [21]. This will lower its internal excitation but, apparently, does not change as much the isotopic composition.

Excited state populations have thermal characteristics [33] and have been shown to correspond to expected temperatures in compound reactions [15,34]. In the present case, the observed internal fragment excitations, associated with the final breakup of the system, are found to be consistent with a thermal population at $T=5$ to $6 \mathrm{MeV}$ (Fig. 4). The apparent temperatures $L_{\mathrm{Li}}$ and $T_{\mathrm{Be} 8-1}(3.04 \mathrm{MeV} / \mathrm{g}$.s.), derived from states not widely separated in energy, are lower but in accordance with the side-feeding effects predicted by the quantum-statistical model [35]. 


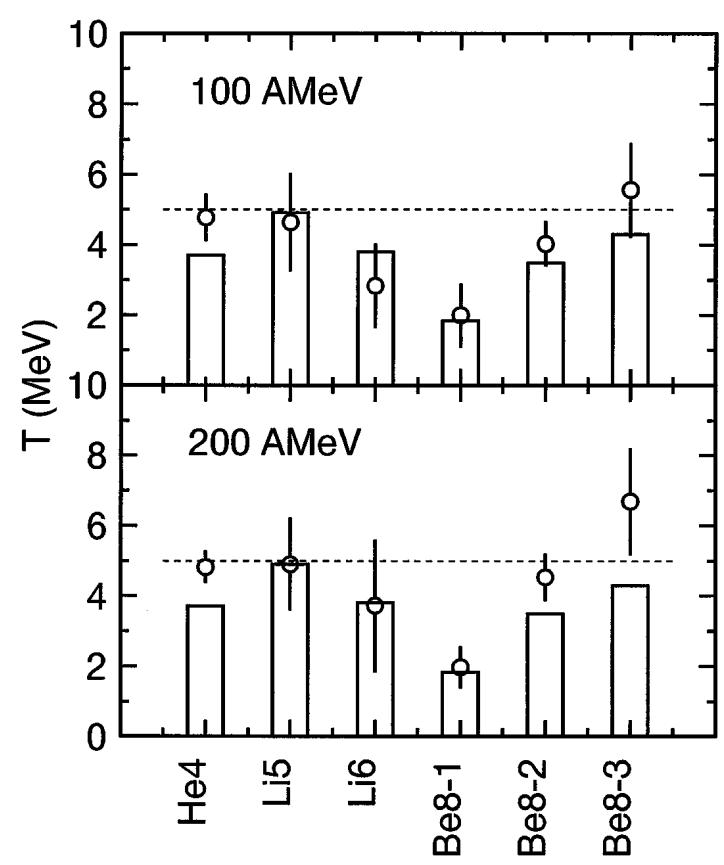

FIG. 4. Six values of apparent excited-state temperatures deduced from states in ${ }^{4} \mathrm{He},{ }^{5} \mathrm{Li},{ }^{6} \mathrm{Li}$, and ${ }^{8} \mathrm{Be}$ (open circles) in comparison to predictions of the quantum-statistical model (bars) for $E / A=100 \mathrm{MeV}$ (top) and $200 \mathrm{MeV}$ (bottom). The calculations were performed with $T=5 \mathrm{MeV}$ (dashed lines) and $\rho / \rho_{0}=0.1$. The notation for $T_{\mathrm{Be} 8}$ is (1) $3.04 \mathrm{MeV} /$ g.s.; (2) $18 \mathrm{MeV} /$ g.s.; and (3) $18 \mathrm{MeV} / 3.04 \mathrm{MeV}$.

The presented interpretation of the observed qualitative difference between the isotope and excited-state temperatures seems rather attractive. It implies that isotope yields may be used to probe the early stages of the fragment formation process, and it may explain the saturation of the excited-state temperatures that characterizes a wide variety of measurements at intermediate and relatiistic energies $[13,15]$. This interpretation, therefore, should be confirmed by further work which may aim at a quantitative interpretation of the internal fragment excitation but also address current open questions such as the role of initial correlations (see, e.g., [21,24,36,37]) and of quantum effects in the fragment formation process [38-41]).

Extensive discussions with J. Aichelin, H.W. Barz, F. Gulminelli, G. Röpke, and M. B. Tsang are gratefully acknowledged. J.P. and M. B. acknowledge the financial support of the Deutsche Forschungsgemeinschaft under Contracts No. Po 256/2-1 and No. Be1634/1-1, respectively. This work was supported by the European Community under Contract No. ERBFMGECT950083.

*Present address: Department of Physics, Yale University, New Haven, CT 06512.

${ }^{\dagger}$ Present address: National Superconducting Cyclotron
Laboratory, Michigan State University, East Lansing, MI 48824.

[1] J. Pochodzalla et al., Phys. Rev. Lett. 75, 1040 (1995).

[2] D. H. E. Gross, Rep. Prog. Phys. 53, 605 (1990).

[3] J. P. Bondorf et al., Phys. Rep. 257, 133 (1995).

[4] Hongfei Xi et al., Z. Phys. A 359, 397 (1997).

[5] Hongfei Xi et al., Phys. Rev. C 54, R2163 (1996).

[6] F. Gulminelli and D. Durand, Nucl. Phys. A615, 117 (1997).

[7] J. B. Natowitz et al., Phys. Rev. C 52, R2322 (1995).

[8] L. G. Moretto et al., Phys. Rep. 287, 249 (1997).

[9] D. J. Morrissey et al., Annu. Rev. Nucl. Part. Sci. 44, 65 (1994).

[10] W. Reisdorf et al., Nucl. Phys. A612, 493 (1997), and references therein.

[11] S. Albergo et al., Nuovo Cimento Soc. Ital. Fis. 89A, 1 (1985).

[12] J. Pochodzalla et al., Phys. Rev. C 35, 1695 (1987).

[13] G. J. Kunde et al., Phys. Lett. B 272, 202 (1991).

[14] H. J. Rabe et al., Phys. Lett. B 196, 439 (1987).

[15] C. Schwarz et al., Phys. Rev. C 48, 676 (1993).

[16] D. H. Boal and J.C. Shillcock, Phys. Rev. C 33, 549 (1986).

[17] V. Serfling, Ph.D. thesis, Universität Frankfurt, 1997 (unpublished).

[18] M. J. Huang et al., Phys. Rev. Lett. 78, 1648 (1997).

[19] The same observation has recently been made for ${ }^{86} \mathrm{Kr}+{ }^{93} \mathrm{Nb}$ central collisions. Hongfei Xi et al. (private communication).

[20] P. Danielewicz and Q. Pan, Phys. Rev. C 46, 2002 (1992).

[21] H. W. Barz et al., Phys. Lett. B 382, 343 (1996).

[22] A. Strachan and C.O. Dorso, Phys. Rev. C 55, 775 (1997).

[23] C. O. Dorso and J. Aichelin, Phys. Lett. B 345, 197 (1995).

[24] R. K. Puri et al., Phys. Rev. C 54, R28 (1996).

[25] J. A. Hauger et al., Phys. Rev. Lett. 77, 235 (1996).

[26] Y.-G. Ma et al., Phys. Lett. B 390, 41 (1997).

[27] H. Xi et al., Report No. MSUCL-1055 (1997).

[28] J. P. Bondorf et al., Nucl. Phys. A296, 320 (1978).

[29] P. J. Siemens and J. O. Rasmussen, Phys. Rev. Lett. 42, 880 (1979).

[30] H. Stöcker et al., Z. Phys. A 303, 259 (1981).

[31] M. Schmidt et al., Ann. Phys. (N.Y.) 202, 57 (1990).

[32] T. Alm et al., Phys. Lett. B 346, 233 (1995).

[33] T. K. Nayak et al., Phys. Rev. Lett. 62, 1021 (1989).

[34] H. Dabrowski et al., Phys. Lett. B 247, 223 (1990).

[35] J. Konopka et al., Phys. Rev. C 50, 2085 (1994).

[36] J. Knoll and J. Wu, Nucl. Phys. A481, 173 (1988).

[37] G. J. Kunde et al., Phys. Rev. Lett. 70, 2545 (1993).

[38] R. Donangelo et al., Phys. Rev. C 50, R563 (1994).

[39] A. Ono and H. Horiuchi, Phys. Rev. C 53, 2958 (1996).

[40] A. Ohnishi and J. Randrup, Phys. Lett. B 394, 260 (1997).

[41] H. Feldmeier and J. Schnack, Prog. Part. Nucl. Phys. 39, 393 (1997). 